\title{
Das Informationsverhalten von Streamern und Zuschauern bei Social Live-Streaming Diensten am Fallbeispiel YouNow
}

https://doi.org/10.1515/iwp-2017-0065

Zusammenfassung: In den letzten Jahren hat eine neue Art von Social Media an Popularität gewonnen, nämlich die Social Live-Streaming Services (SLSSs). Ein besonders bei Jugendlichen und jungen Erwachsenen beliebter SLSS ist YouNow, der auch im Fokus unserer Fallstudie steht. SLSSs ermöglichen dem Nutzer in Echtzeit zu streamen, mit den Zuschauern zu interagieren und von diesen auf verschiedene Weisen belohnt zu werden. In dieser Studie wurde das Informationsverhalten der Nutzer von SLSSs unter Anwendung sowohl der Lasswell Formel als auch des Nutzen- und Belohnungsansatzes (Uses and Gratification Theory) analysiert. Dabei werden fünf Forschungsfragen beantwortet: Aus welchen Motiven verwenden die Zuschauer und Streamer YouNow? Welches Informationsproduktionsverhalten zeigen die Nutzer? Welche Rechtsverstöße lassen sich während des Streamings beobachten? Welches Multi-ChannelVerhalten weisen Nutzer von YouNow auf? Und, wie bewerten sie die Qualität von YouNow? Um Antworten auf diese Fragen zu erhalten, wurde zum einen eine Online Umfrage ( $\mathrm{N}=123)$ durchgeführt und zum anderen Echtzeitübertragungen systematisch beobachtet $(\mathrm{N}=2.021)$.

Schlagworte: Social Live-Streaming Dienst, Social Networking Dienst, Informationsverhalten, Motiv, Social Media, Nutzer, YouNow, Video

\footnotetext{
*Kontaktperson: Katrin Scheibe, Heinrich-Heine-Universität Düsseldorf, Abteilung für Informationswissenschaft, Universitätsstraße 1, 40225 Düsseldorf,

E-Mail: katrin.scheibe@hhu.de

Franziska Zimmer, Heinrich-Heine-Universität Düsseldorf, Abteilung für Informationswissenschaft, Universitätsstraße 1, 40225 Düsseldorf, E-Mail: franziska.zimmer@hhu.de

Kaja J. Fietkiewicz, Heinrich-Heine-Universität Düsseldorf, Abteilung für Informationswissenschaft, Universitätsstraße 1, 40225 Düsseldorf, E-Mail: kaja.fietkiewicz@hhu.de
}

\author{
Information Behavior of Streamers and Viewers on Social \\ Live Streaming Services: YouNow as a Case Study
}

Abstract: In the last few years a new form of social media has emerged and gained popularity, namely Social LiveStreaming Services (SLSSs). YouNow - our case study - is an SLSS, which is especially popular among adolescents and young adults. SLSSs grant their users the opportunity to broadcast themselves in real time, interact with the viewers and even receive some form of reward from the viewers. A theoretical framework for investigating the information behavior on SLSSs was employed by using the Lasswell Formula as well as the Uses and Gratification Theory. Accordingly, five research questions are being answered in this paper: What motives do viewers and streamers display to apply YouNow? What information production behavior do the users exhibit on YouNow? What law infringements can be observed while streaming? What multi-channel behaviors do users of YouNow exhibit? How do users rate the quality of YouNow? To answer these questions an online survey $(\mathrm{N}=123)$ was implemented and live streams $(\mathrm{N}=2,021)$ were systematically examined.

Keywords: Social Live Streaming Services, Social Networking Services, Information Behavior, Motives, Social Media, Users, YouNow, Video

Le comportement informationnel des streamers et des spectateurs sur des Social Live Streaming Services à partir de l'exemple YouNow

Résumé: Au cours des dernières années, un nouveau type de médias sociaux a gagné en popularité les Social Live Streaming Services (SLSS). Un SLSS qui est particulièrement populaire parmi les jeunes et les jeunes adultes est YouNow, qui fait également l'objet de notre étude de cas. Les SLSS permettent à l'utilisateur le streaming en temps réel et ils lui offrent la possibilité d'interagir avec les spectateurs et d'être récompensé de diverses façons. Cette étude analyse le comportement informationnel des utilisa- 
teurs des SLSS en employant la formule Lasswell, ainsi que l'approche utilisations et récompenses (Uses and Gratification Theory). Cette approche répond à cinq questions de recherche: Pour quels motifs des spectateurs et des streamers utilisent-ils YouNow ? Quel comportement de production d'information les utilisateurs montrent-ils ? Quelles violations de droit peuvent être observées lors de la diffusion? Quel comportement multi-canal les utilisateurs de YouNow ont-ils ? Et comment évaluent-ils la qualité de YouNow ? Afin d'obtenir des réponses à ces questions, un sondage en ligne a été effectué $(\mathrm{N}=123)$, ainsi qu'une surveillance systématique des transmissions en temps réel $(\mathrm{N}=2,021)$.

Descripteurs: Social Live Streaming Services, Service de Réseautage Social, Comportement d'Information, Motif, Médias Sociaux, Utilisateur, YouNow, Vidéo

\section{Einleitung}

Seitdem es im Internet Social Media-Dienste gibt, hat die Menschheit das Bedürfnis, das eigene Leben mit der Welt zu teilen. Nahezu jeder mit einem Internetanschluss ist bei mindestens einem Social Media-Service vertreten. Es ist also kein Wunder, dass Dienste wie WhatsApp und Facebook weit verbreitet zum alltäglichen Mittel der Konversation werden. Sei es ein netter Abend mit Freunden oder doch etwas Interessanteres, wie die nächste Safari bei einer lange geplanten Afrika Reise - schnell ein Foto gemacht und hochgeladen, schon können Freunde und Familie an dem Ereignis teilhaben (Friedländer, 2017b).

In den letzten Jahren hat sich daher eine neue Art von Social Networking Service (SNS) entwickelt: die Social Live-Streaming Dienste (Social Live-Streaming Services, SLSSs). Hier teilt jeder Nutzer seine Erlebnisse über eine Echtzeit-Videoübertragung mit der Welt, sei es über die Webcam eines Computers / Notebooks oder über die Kamera eines Smartphones / Tablets. Die Zuschauer können über ein Nachrichtenfenster mit dem sendenden Nutzer interagieren und diesen sogar mit Aufmerksamkeiten belohnen (Friedländer, 2017a).

Live-Übertragungen im Fernsehen sind nichts Neues; genauso wie die menschliche Schwäche für Reality Shows, kritisch thematisiert im amerikanischen Film „Die Truman Show", in dem der Protagonist, Truman Burbank, unwissentlich 30 Jahre lang rund um die Uhr von Kameras begleitet wird. Mit den Social Live-Streaming Services wie YouNow, Periscope oder Ustream kann jeder Webnutzer zu „Truman” werden und seine Zuseher mit einer Live-Show unterhalten, mit dem essentiellen Unterschied, dass sich die Akteure vor der Kamera stets ihrer Zuschauer bewusst sind (Fietkiewicz \& Scheibe, 2017).

\section{Theoretischer Rahmen}

Anders als bei anderen, asynchronen, Social Networking Services wie z. B. Facebook (Khoo, 2014, S. 81), handelt es sich bei Social Live-Streaming Services um synchrone Dienste. Dies bedeutet, dass alle miteinander verbundenen Aktionen (fast) zeitgleich und unverzüglich aufeinander folgend stattfinden. Außerdem kann bei Social LiveStreaming Diensten zwischen zwei Service-Arten unterschieden werden. Zum einen gibt es allgemeine Live-Streaming Dienste ohne thematischen Schwerpunkt (z. B. YouNow, Periscope, Ustream, Facebook Live, YouTube Live oder Google Hangouts), zum anderen themenbezogene Live-Streaming Dienste wie Twitch für Videospiel-Übertragungen und -Events (Gros et al., 2017) oder Picarto im Bereich Kunst (Scheibe, Fietkiewicz, \& Stock, 2016).

Die Nutzer eines Social Media Netzwerks produzieren und konsumieren eine Vielzahl an Informationen (O'Reilly \& Battelle, 2009). Toffler (1980) führte daher den Begriff „Prosumer“ ein, der sich aus dem englischen „Producer“ und „Consumer“ zusammengesetzt. Scheibe, Fietkiewicz und Stock (2016) haben das Informationsverhalten von Produzenten und Konsumenten in einem Social Live-Streaming Dienst anhand eines Modells beschrieben. In Abbildung 1 wurde das Modell um die Lasswell-Formel (Lasswell, 1948) sowie den Nutzen- und Belohnungsansatz (Uses and Gratification Theory) von Blumler und Katz (1974) und andere theoretische Aspekte erweitert.

Ausgehend von der Kommunikationstheorie von Harold D. Lasswell (1948, S. 37) und der damit verbundenen Frage: Wer sagt was in welchem Kanal zu wem mit welchem Effekt? (,Who says what in which channel to whom with what effect?"), wird von Lasswell - ausgehend vom Produzenten - ein Prozess beschrieben, der auch in unserem Modell wiederzufinden ist. Nutzer X (,Wer sagt?”) produziert eine Publikation mit einem bestimmten Inhalt, auf YouNow ist es ein Livestream (,Was?”), und verteilt sie im Social Networking Service („In welchem Kanal?”). Nutzer Y („Zu Wem?”) findet und betrachtet den Livestream, erhält daraus Informationen und schreibt dem Nutzer eine Nachricht oder belohnt diesen sogar mit einer Aufmerksamkeit („Mit welchem Effekt?”). Aber warum verwenden Nutzer einen Social Networking-Dienst und produzieren oder konsumieren bestimmte Inhalte?

Basierend auf dem Nutzen- und Belohnungsansatz von Blumler und Katz (1974) ist die Verwendung von Medien durch die Erfüllung bestimmter Bedürfnisse und dem 


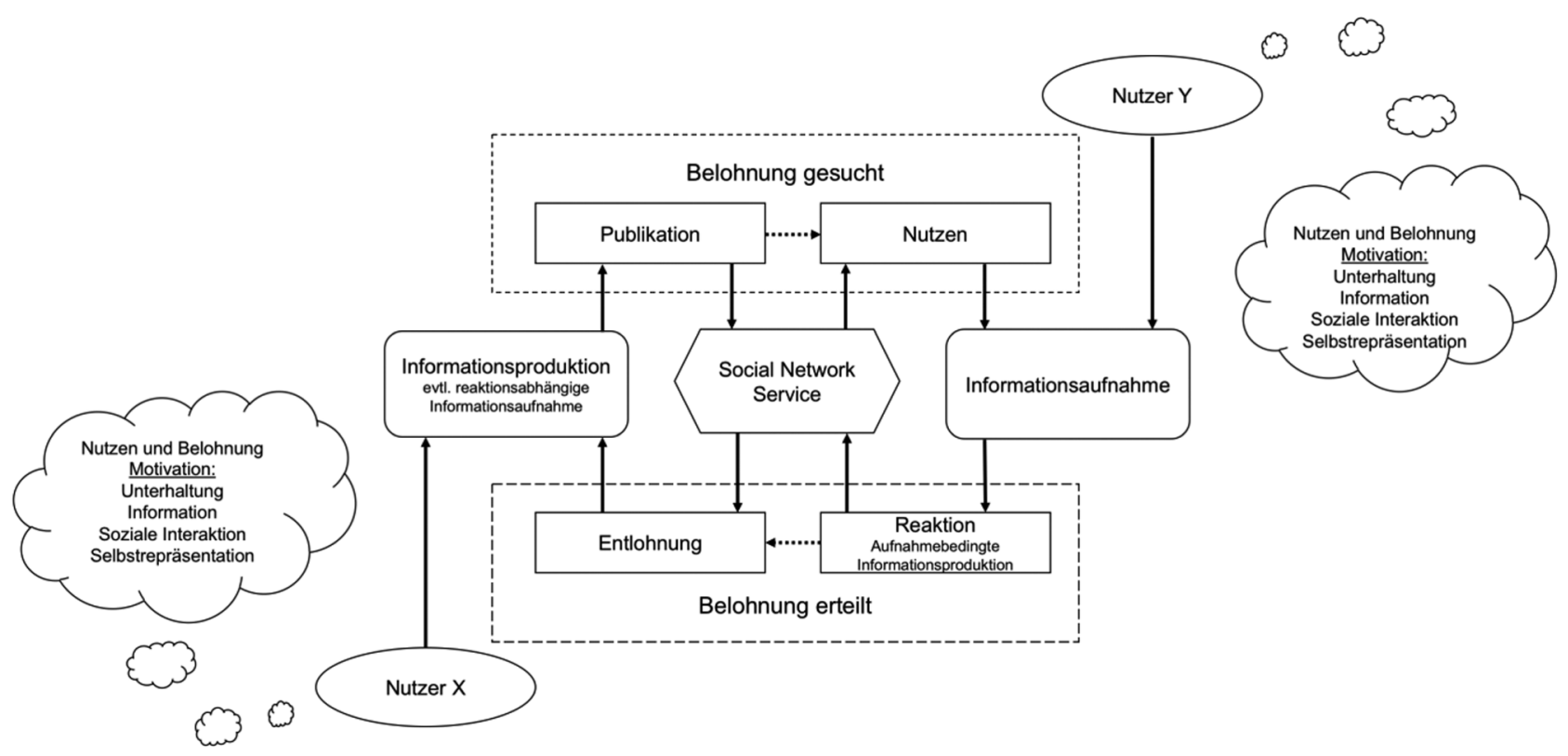

Abbildung 1: Informationsverhalten von Produzenten und Konsumenten in einem Social Network Service.

Suchen nach Belohnung und Genugtuung geleitet. Zudem ist die Verwendung von Medien von gewissen Erwartungen geprägt (Katz, Blumler \& Gurevitch, 1973) und zielgerichtet (McQuail, Blumler, \& Brown, 1972). Es gibt verschiedene Gründe, die Nutzer dazu veranlassen, Medien zu verwenden. McQuail (1983) nennt vier zentrale Punkte: Unterhaltung, Information, Selbstverwirklichung und soziale Interaktion. Shao (2009) unterscheidet verschiedene Motive in Bezug auf die vom Nutzer ausgeführte Handlung: konsumieren, teilhaben oder produzieren. Konsumenten, also Nutzer, die nur lesen oder zuschauen ohne am Geschehen teilzunehmen, nutzen Social Media-Dienste vorwiegend zur Information und Unterhaltung. Das Ziel von Teilnehmern, also Nutzern, die konsumieren und reagieren bzw. am Geschehen teilnehmen, ist zusätzlich die soziale Interaktion. Produzenten erstellen Livestreams und reagieren auf Beiträge von anderen Nutzern und haben (wiederum zusätzlich) Selbstdarstellung und Selbstverwirklichung als Ziele.

Palmgreen, Wenner und Rayburn (1980) diskutieren die Aspekte von „Belohnung (Genugtuung) gesucht“ und „Belohnung (Genugtuung) erhalten“ in Zusammenhang mit dem Nutzen- und Belohnungsansatz. Eine Genugtuung, die gesucht wird, wird - bei optimal verlaufender Kommunikation - in einer gewissen Weise erreicht. Auch beziehen sich Nutzen und Belohnung auf verschiedene Formen von Informationsproduktions- sowie Informationsempfangsverhalten.

In Abbildung 1 suchen Benutzer X und Benutzer Y nach Genugtuung durch Unterhaltung, Information, sozialer Interaktion und Selbstdarstellung. Der Social Live-
Streaming Service YouNow bietet den Zuschauern die Möglichkeit, als Gast in einem Livestream teilzunehmen, was bei den Teilnehmern zusätzlich die Motive von Produzenten aufweist. Produzenten interagieren live vor der Kamera mit den Zuschauern über den Live-Chat. Streamer lesen die Chat-Nachrichten (konsumieren) und können augenblicklich antworten (Teilnahme). Folglich haben sie (auch) die gleichen Motive wie Konsumenten oder Teilnehmer. In dem Modell werden Gratifikationen durch das Publizieren (Livestreaming) sowie durch das Nutzen gesucht. Die Informationsaufnahme des Zuschauers führt (im positiven Fall) zur Genugtuung des Benutzers Y. Wenn Benutzer Y eine Reaktion auf den Livestream von Benutzer $\mathrm{X}$ zeigt, in Form einer Chatnachricht oder einer Aufmerksamkeit, hat auch $\mathrm{X}$ (wiederum im positiven Fall) seine gesuchte Belohnung erhalten.

Basierend auf dem Modell aus Abbildung 1 werden in dem vorliegenden Artikel folgende Forschungsfragen beantwortet:

- Welche Motive haben Zuseher und Streamer, um YouNow zu verwenden?

- Welches Informationsproduktionsverhalten zeigen die Streamer auf YouNow auf?

- Welche Rechtsverstöße können während des Streamings auf YouNow beobachtet werden?

- Welches Multi-Channel-Verhalten weisen Nutzer von YouNow auf?

- Wie bewerten Nutzer die Qualität von YouNow? 


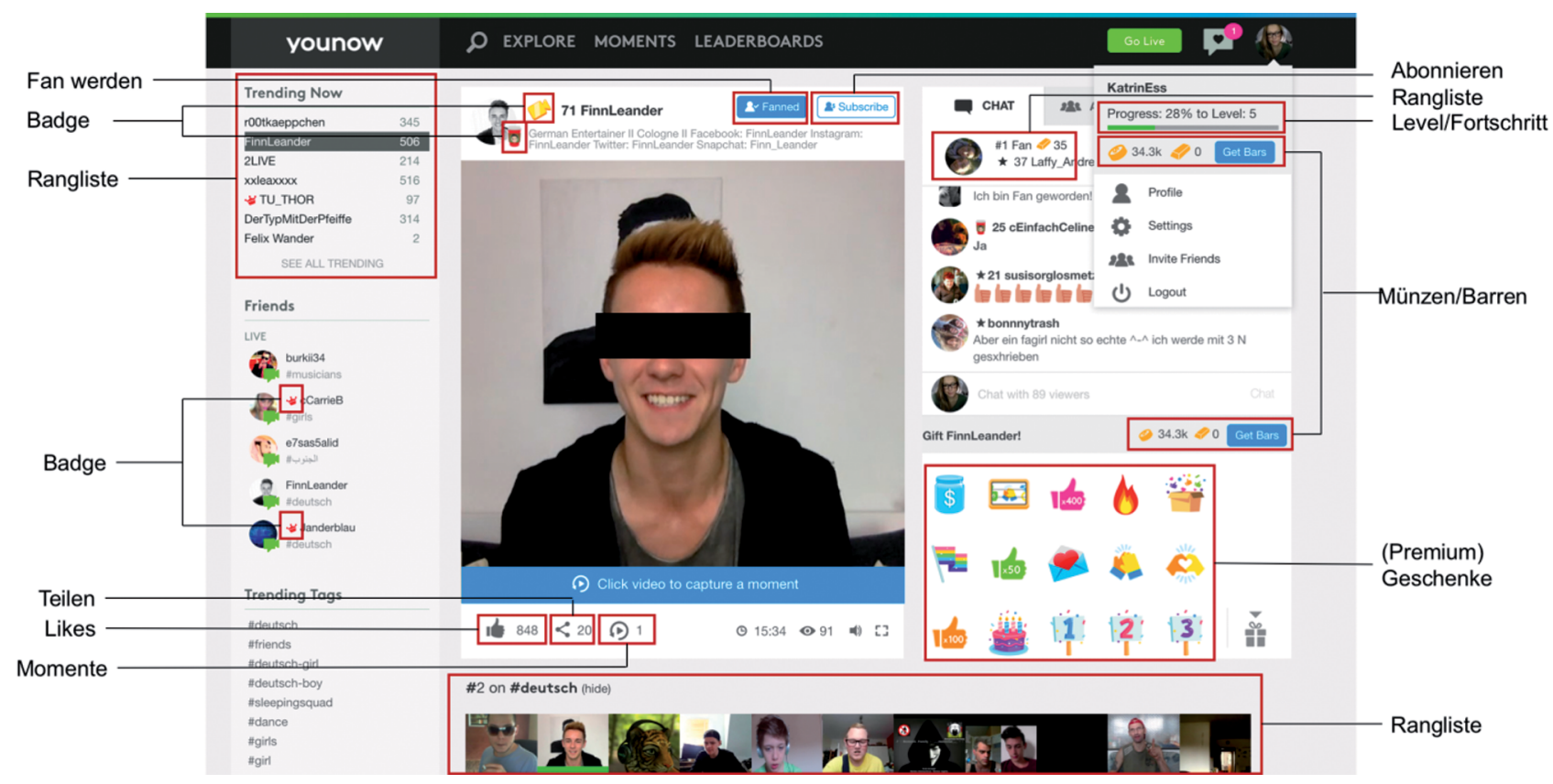

Abbildung 2: YouNow Livestream.

\section{YouNow als Fallbeispiel}

„YouNow ist der beste Weg, um talentierte Broadcaster zu entdecken, Live-Streams zu schauen und um in Echtzeit mit Leuten aus der ganzen Welt zu kommunizieren" (YouNow, 2016). Der Social Live-Streaming Service YouNow wurde ursprünglich dazu entwickelt, dass YouTuber mit ihren Fans in Kontakt treten, live mit ihnen kommunizieren und deren Fragen beantworten. Viele Teenager und junge Erwachsene hatten Spaß an der Verwendung und den Funktionen, teilten ihre Erfahrungen mit dem System mit Freunden und fingen an, ihre eigenen Fangemeinschaften auf YouNow aufzubauen. Adi Sideman, dem Erfinder und CEO von YouNow, zufolge werden rund 150.000 Livestreams täglich gesendet (2015).

Um auf die Seite von YouNow zu kommen, muss man bestätigen, dass man 13 Jahre oder älter ist. Nach Abruf der Seite startet sofort ein laufender Livestream. Man kann sich mit einem Account bei Facebook, Twitter, Instagram oder Google+ auf YouNow einloggen, nicht aber mit einer E-Mail-Adresse. Nur durch die Anmeldung ist es möglich, Chatnachrichten bei laufenden Livestreams zu schreiben, den Nutzern Aufmerksamkeiten zu schicken oder selbst einen Livestream zu starten. Dem Livestream kann ein Hashtag zugeordnet werden, über den das Video von anderen Nutzern zu finden ist. Zusätzlich kann man seinen eigenen sowie auch den Livestream anderer Nutzern auf anderen Social Media-Seiten ankündigen und teilen. Wie man in Abbildung 2 sieht, ist der Livestream in der Mitte der Webseite platziert. Direkt darunter findet man zum einen die Anzahl, wie oft jemandem der Livestream gefallen hat, und zum anderen, wie oft der Livestream geteilt wurde. Zusätzlich erfährt der Nutzer, wie lange der Livestream schon läuft und wie viele Zuschauer zu dem Zeitpunkt zusehen. Oberhalb des Livestreams findet man das Profilbild, ein Badge, das Level und den Benutzernamen. Das Badge sowie das Level zeigen an, wieviel Erfahrung der Streamer mit dem Senden von Livestreams hat. Zudem sind oben zwei Felder, um Fan von dem sendenden Nutzer $\mathrm{zu}$ werden und um den Nutzer zu abonnieren. Auf der linken Seite findet man eine Rangliste mit den derzeit beliebtesten Live-Übertragungen, eine Liste mit Freunden, die momentan einen Livestream senden, sowie eine Liste mit beliebten Hashtags. Auf der rechten Seite sind das Chatfenster und eine Auflistung von Geschenken platziert, die man dem Streamer zusenden kann. Außerdem gibt es verschiedene Tabs, mit denen man zwischen Chatfenster, aktuellen Zuschauern und Gast-Anfragen wechselt.

Die YouNow-Community verteilt Aufmerksamkeiten im Chat, diese können zum Beispiel Sticker, Emojis, Icons und Likes sein (rechts in Abbildung 2). Damit zeigen die Nutzer nicht nur ihre Aufmerksamkeit und ihr Interesse an dem Broadcaster, sondern wollen damit zusätzlich selbst auffallen. Um ein Geschenk oder eine Aufmerksamkeit zu verschicken, muss man Münzen, die virtuelle Währung auf YouNow, sammeln. Münzen sammelt man durch verschiedene Webseiten-Aktivitäten, beispielsweise durch das Online Gehen, durch das Streamen und durch das Chatten. 
Eine andere Währung, die mit echtem Geld gekauft werden muss, heißt „Bars“. Bars ermöglichen es den Nutzern bestimmte Premium-Geschenke zu kaufen. Die Anzahl der Münzen und Barren, die man aktuell besitzt, werden unter dem Chatfenster angezeigt. Jeder Nutzer weist ein Level auf, das durch verschiedene Aktivitäten, wie das Senden oder Zuschauen von Streams oder auch durch das Einbinden anderer Social Media Accounts, steigt. Je höher das Nutzer-Level, desto mehr Geschenke und Besonderheiten werden auf der Seite freigeschaltet. Am Ende eines Livestreams bekommt der Streamer verschiedene statistische Werte über das Video angezeigt. Man kann sehen, wie viele Likes man bekommen hat, wie viele Nutzer zugeschaut haben, wie viele Münzen man verdient hat und wie viele Fans man dazu bekommen hat.

Nach der Web-Statistik Seite Alexa (Daten für 2016) kommen die meisten Seitenbesucher von YouNow aus den Vereinigten Staaten von Amerika (24,4\%), der Türkei (11,4\%), Mexiko (10,1\%), Deutschland (8,6\%) und SaudiArabien (4,9\%). Am liebsten (weit über dem Internetdurchschnitt) greifen die Nutzer auf YouNow von zu Hause aus zu. Auch leicht über dem Internetdurchschnitt sind die Aufrufe aus der Schule, wohingegen Seitenbesuche vom Arbeitsplatz aus leicht unter dem Internetdurchschnitt liegen. Viele Nutzer von YouNow gehen noch zur Schule und nur einige wenige von ihnen sind berufstätig. Ausgehend von unseren Umfragedaten wird YouNow hauptsächlich von Teenagern und jungen Erwachsenen zwischen dem 13. und 22. Lebensjahr genutzt, die meisten Umfrageteilnehmer waren 16 Jahre alt.

\section{Rechtsverletzungen}

In dem folgenden Abschnitt werden mögliche Rechtsverletzungen und oft missachtete Geschäftsbedingungen näher erläutert und es wird erklärt, wie sie bei der Nutzung von Social Live-Streaming Diensten wie YouNow auftreten können. Für diese Untersuchung wurde das deutsche Recht gewählt, da es das strikteste der drei untersuchten Länder Deutschland, Japan und USA ist. Eine Ausnahme wurde bei dem Urheberrecht bezogen auf Musik gemacht. Hier wurde der Aspekt von „fair use“ des amerikanischen Rechts oder das „Beiwerk“ des deutschen Rechtes verwendet und großzügig ausgelegt. Das deutsche Gesetz über das Urheberrecht und verwandte Rechte besagt, dass der Autor das Recht hat $\mathrm{zu}$ bestimmen, falls und wie seine Arbeit veröffentlicht werden soll. Weiterhin hat der Autor das exklusive Recht der Reproduktion, das Recht der Verbreitung und das Recht der Ausstellung seines Werkes, auch in nicht-materieller Form, was auch das Übertragen von
Streams beinhaltet (Urheberrecht § 15(II), § 20). Demnach muss der Streamer das explizite Recht erhalten haben, urheberrechtlich geschützten Inhalt in seinem Stream verwenden zu dürfen. Eine Ausnahme hiervon ist das Berichten über aktuelle Events und die Reproduktion und öffentliche Verbreitung dieser Nachrichten (Urheberrecht § 50). Es ist weiterhin gestattet, intellektuelles Eigentum zu zitieren (Urheberrecht $\S 51$ ). Für diese Studie wurde Musik, die im Hintergrund spielt, als Beiwerk (Urheberrecht $\S 57$ ) bewertet. In Amerika ist es erlaubt, urheberrechtlich geschütztes Material unter der Regelung des „fair use“ (§ 107 U.S. Copyright) zu verwenden. Musik wurde nur als Urheberrechtsverletzung gewertet, wenn die Musik deutlich der Inhalt des Streams war. Verletzungen des Urheberrechts sind ein großes Problem im Internet und auch bei SLSSs (Rugg \& Burroughs, 2016). Es kann argumentiert werden, dass die Verletzungen des Urheberrechts negative Konsequenzen haben sollten; jedoch könnten sie auch dabei helfen, ein Produkt populärer zu machen und sogar die Anzahl der Verkäufe zu steigern (Linde \& Stock, 2011).

Die Persönlichkeitsrechte sind in Artikel 1 und Artikel 2 des Deutschen Grundgesetzes gesichert und besagen, dass die menschliche Würde unantastbar ist. Die Persönlichkeitsrechte sind zudem durch $\S 823$ (I) des Bürgerlichen Gesetzbuches (BGB) geschützt. Das bedeutet weiterhin, dass eine Person das Recht hat, nicht gefilmt oder in der Öffentlichkeit gestreamt zu werden, auch wenn die Person sich nur im Hintergrund befindet. Wenn so ein Fall beobachtet wurde, wurde dies als Verletzung des Persönlichkeitsrechtes markiert. Weitere Kategorien der Persönlichkeitsrechte wurden gesondert betrachtet. Dies sind Datenschutz und Beleidigungen. Nach $\S 1$ des Bundesdatenschutzgesetzes (BDSG) hat jede Person das Recht, gegen Verletzungen der Privatsphäre aufgrund von falschen Handhabungen der persönlichen Daten beschützt zu werden. Dies beinhaltet die Weitergabe von Informationen über die Adresse einer Person, ihre Telefonnummer oder das Lesen von persönlichen Nachrichten ohne die ausdrückliche Zustimmung der betroffenen Person. Streamer unterhalten sich über verschiedenste Themen. Daher ist es wahrscheinlich, dass auch persönliche Daten weitergegeben werden. Beleidigungen werden mit einer Gefängnisstrafe von bis zu einem Jahr oder einer Geldstrafe geahndet, wie es in $\S 185$ des Strafgesetzbuches (StGB) steht. Beleidigungen geschehen im täglichen Leben und im Internet und sind sicherlich auch in Streams zu beobachten. Diese potentielle Rechtsverletzung muss jedoch im Kontext des Streams betrachtet werden. Denn eine Beleidigung muss nicht unbedingt so gemeint sein, nur weil ein potentiell abwertendes Wort verwendet wurde, denn dies ist situationsabhängig. Zum Beispiel können mögliche Be- 
schimpfungen zwischen Freunden in einem spaßig gemeinten Kontext verwendet und demnach nicht als Beleidigung aufgefasst werden.

Die Straßenverkehrsordnung betreffend ist es nicht erlaubt, ein Handy oder ein Autotelefon in der Hand zu halten, während das Fahrzeug bedient wird. Die einzige Ausnahme besteht dann, wenn das Fahrzeug steht und der Motor ausgeschaltet ist (§ 23 der Straßenverkehrsordnung). Da YouNow auf einem Handy verwendet werden kann, ist es möglich, dass die Straßenverkehrsordnung missachtet wird.

Ein Gesetz regelt die Übertragung von kommerziellen Sportereignissen zwar nicht, jedoch gibt es zugehörige Regeln und Gesetze, die angewandt werden können. Es gibt allerdings auch eine Reihe von Gegenargumenten, weswegen diese Gesetze nicht für Sportereignisse gelten (Pötters, 2011); diese werden hier jedoch nicht aufgeführt. Edelmann (2015) beschreibt, dass die Sportindustrie durch Live-Streaming Verluste erleiden könnte. Für unsere Studie wurden Sportübertragungen dann als Rechtsverletzung markiert, wenn das Ereignis entweder mit einem Handy aus einem Stadion oder von einem Fernseher abgefilmt wurde. Wenn eine Person zum Beispiel ein Bundesligaspiel von Borussia Mönchengladbach besucht, könnte diese das Spiel mit dem Handy streamen. Die folgenden Gesetze müssten dann möglicherweise angewandt werden. Nach $\S 4$ des deutschen Gesetzes gegen unlauteren Wettbewerb (UWG) verhält sich jemand unfair, wenn er den Service eines anderen imitiert und ihn für seinen eigenen Nutzen verwendet oder den Gewinn des Besitzers beeinträchtigt. Der Veranstalter des Events könnte unfair beeinträchtigt werden, da er nicht in der Lage ist, es für den gleichen kommerziellen Gewinn zu nutzen, wie wenn es keine Übertragung des Events durch Dritte geben würde (§ 3 UWG). Weiterhin hat jeder Veranstalter das Recht, sein Hausrecht zu nutzen und das Streamen von Ereignissen zu untersagen (Bürgerliches Gesetzbuch § 823 Abs. 1, § 1004, $\S 862)$. Dies gewährt Veranstaltern die Sicherheit, die kommerziellen Einnahmen in vollem Maße zu erhalten. Wenn die Person eine Sportübertragung von einem Fernseher mit dem Handy streamt, wird dies als Urheberrechtsverletzung markiert ( 87 UrhG), denn die Person streamt geistiges Eigentum des TV-Senders. Dieselben Regeln lassen sich jedoch nicht für den Amateursport anwenden (Bundesgerichtshof, D. f. 10.28.2010 - I ZR 60/09), da hier der kommerzielle Aspekt fehlt.

Der Health Insurance Portability and Accountability Act (HIPAA) des amerikanischen Gesetzes reguliert die Nutzung von Informationen über den Gesundheitsstatus einer Person. Diese Art von Information ist automatisch im Datenschutzgesetz in Deutschland gesichert, jedoch haben wir uns dazu entschieden, diese potentiellen Verletzungen gesondert zu betrachten, da Periscope und vielleicht auch YouNow dafür verwendet werden, um Operationen live zu übertragen.

Das Mindestalter wird nicht durch das Gesetz geregelt, sondern durch jeden Anbieter selbst. YouNow setzt das Mindestalter auf 13 Jahre.

\section{Methoden}

Um unsere Forschungsfragen beantworten zu können, wurden zwei empirische Untersuchungen durchgeführt, eine Online-Umfrage und Beobachtungen von Echtzeitübertragungen. Die Umfrage fand vom 3. bis 28. Juni 2015 mit 123 YouNow-Nutzern auf Umfrageonline.com statt. Die Echtzeitübertragungen wurden vom 26. April 2016 bis 24. Mai 2016 beobachtet und umfassen 2.021 Streams. Der Großteil der Teilnehmer kam aus Deutschland oder den Vereinigten Staaten von Amerika. Zusätzlich wurden vor der systematischen Beobachtung diverse Livestreams verfolgt, um Kategorien für eine Inhaltsanalyse des Contents und für die Motive der Streamer zu gewinnen.

Die Beschreibung und Analyse von Motiven und Inhalten erfordert das Vorliegen von passenden Kategorien denen die jeweiligen Streams zuzuordnen sind. Vier Studien setzen sich mit den Motiven der Streamer und den nutzergenerierten Inhalten in SLSSs auseinander: Scheibe, Fietkiewicz und Stock (2016), Tang, Veniola und Inkpen (2016) und Friedländer (2017a, 2017b) zeigen auf, warum Streamer auf SLSSs aktiv werden. Weiterhin konnten mehrere Studien über Motive und das Verhalten von Nutzern auf anderen SNSs wie Twitter und Facebook ausgemacht werden.

Zusätzlich zu der Literaturrecherche wurden Videos der SLSSs analysiert, um passende Kategorien für die Inhalte der Streams und die Motive der Nutzer festzulegen. Diese lassen sich nach dem Nutzen- und Belohnungsansatz klassifizieren. Die Oberbegriffe sind Unterhaltung, soziale Interaktion, Informationssuche und Selbstinszenierung (Hsu, Chang, Lin, \& Lin, 2015). Nach diesen Aspekten lassen sich auch die in der Literatur gefundenen Motive einordnen. Jene, die sich dem Bedürfnis nach Unterhaltung zuordnen lassen, sind unter anderem die Langeweile (Brandtzag \& Heim, 2009) oder auch Spaß (Cheung, Chiu, \& Lee, 2011; Kim, Kim, \& Nam, 2010). Soziale Interaktion wird betrieben, um Einsamkeit vorzubeugen oder dieser zu entgehen, wie dies auf Facebook und Twitter beobachtet wurde (Brandtzaeg \& Heim, 2009). Auch bereits bestehende Beziehungen und soziale Aktivitäten werden via soziale Medien gepflegt (Beldad \& Koehorst, 2015; Tosun, 2012). 
Soziale Interaktionen innerhalb einer virtuellen Gemeinschaft erfüllen das Bedürfnis des Nutzers dazuzugehören und erhöhen das gruppenbasierende Selbstbewusstsein (Cheung et al., 2011). Auch wenn noch keine sozialen Strukturen bestehen, hat jeder Nutzer die Möglichkeit, seine eigene Gemeinschaft aufzubauen (Hollenbaugh \& Ferris, 2013). Wenn es um die Informationssuche geht, finden sich Motive wie der Meinungsaustausch oder die Verbreitung der eigenen Meinung (Lin \& Lu, 2011). SNSs bieten die Möglichkeit, spezielle Gruppen zu erreichen, um mit ihnen zu interagieren (Joinson, 2008) und Informationen auszutauschen. Auch Organisationen verwenden SNSs als wichtiges Kommunikationswerkzeug (Kim, Kim, \& Nam, 2014). Die Selbstinszenierung zeigt Motive, wie das Bedürfnis berühmt zu werden oder Geld durch den Gebrauch von SNSs zu generieren; die Nutzer wollen gesehen und akzeptiert werden (Greenwood, 2013). Marwick und Boyd (2010; 2011) beschreiben das Phänomen eines Nutzers, der im Kontakt mit seiner Gemeinschaft bleiben will, um seine Persönlichkeit als „Micro-Celebrity“ aufzubauen und als „Influencer“ zu vermarkten. Das Sendungsbewusstsein spielt auch eine große Rolle im Gebrauch von SNSs (Beldad \& Koehorst, 2015; Greenwood, 2013). Zusammengefasst haben wir basierend auf der Literaturrecherche und unseren eigenen Beobachtungen die folgenden Motive mit ihren Oberbegriffen formuliert:

- Unterhaltung: Langeweile, Spaß, Hobby

- Soziale Interaktion: Einsamkeit, Beziehungsmanagement, Bedürfnis nach Kommunikation, Bedürfnis dazuzugehören

- Selbstinszenierung: Selbstverbesserung, Sendungsbewusstsein, ein Star werden, Geld verdienen, Troll

- Informationssuche: eine spezielle Gruppe erreichen, Meinungen austauschen

- Kein Kommentar

Mit der gleichen Methode haben wir Kategorien definiert, die den Inhalt der Videos beschreiben. Menschen nutzen SNSs, um zu kommunizieren (Beldad \& Koehorst, 2015; Brandtzaeg \& Heim, 2009). Dies gilt aller Wahrscheinlichkeit nach auch für SLSSs. Hier teilen Personen Informationen über verschiedene Themen oder über sich selbst (Hollenbaugh \& Ferris, 2013). Das könnte zum Beispiel ein Tutorial in Form eines Fitnessvideos sein. Unterhaltung ist ein weiterer Faktor, um SNSs zu nutzen (Cheung, Chiu, \& Lee, 2011; Kim, Sohn, \& Choi, 2010). Dafür werden verschiedene Arten von Unterhaltungsmedien verwendet. Livestreams bieten die Möglichkeit, Filme, TV Serien oder Musik zu übertragen. Die Medien können sogar 24 Stunden am Tag, sieben Tage die Woche gesendet werden. Daher wurde die Kategorie 24/7 definiert. Auch das Strea- men von Videospielen ist eine Form der Unterhaltung. Twitch.tv ist bereits die Hauptplattform, um Gaming-Videos und eSport zu übertragen. Aber Gamer könnten auch YouNow verwenden. Beispiele wie Justin Bieber und Lindsey Stirling zeigen deutlich, dass Musiker, die auf YouTube begonnen haben, sehr populär werden können. Es ist also plausibel, dass Streamer eine Chance darin sehen, sich beim Musizieren zu filmen und dadurch ihren Bekanntheitsgrad zu steigern. Eine Studie von Tang, Venolia und Inkpen (2016) definierte verschiedene Kategorien für den Inhalt auf SLSSs. Einige der Kategorien wurden für unsere Forschungsarbeit übernommen. Dies bezieht Menschen mit ein, die sich beim Essen zu Hause oder in einem Restaurant filmen und das streamen. Eine andere Kategorie von Tang et al. ist das Filmen von Tieren, entweder zu Hause oder in der Natur. Auch Natur an sich wurde als eine Kategorie definiert. Einen anderen Aspekt, den Tang et al. (2016) nannten, ist das Filmen und Streamen einer speziellen Fähigkeit, wie zum Beispiel das Malen eines Bildes oder die zur Schaustellung eines komödiantischen Talents. Auch der Alltag eines Streamers wird gefilmt, zum Beispiel während er schläft, zur Arbeit geht oder seine Zähne putzt. SLSSs können außerdem für wissenschaftliche Zwecke verwendet werden, zum Beispiel um Informationen über Wissenschaft, Technik und Medizin zu vermitteln. Rugg und Burroughs (2016) beschreiben, dass Leute streamen, um Nachrichten zu verbreiten. SNSs werden verwendet, um politische Themen $\mathrm{zu}$ diskutieren (Hampton, Shin, \& Lu, 2016), es wäre also keine Überraschung, wenn auch SLSSs dafür verwendet werden. Da SLSSs live sind und der Inhalt in Echtzeit generiert wird, wurde auch die Kategorie „Nichts“ definiert. Dies bedeutet, dass kein Inhalt produziert wurde, als die Daten gesammelt wurden. SLSSs erlangen mehr und mehr Popularität. Das bedeutet, dass sie das Potential bieten, durch sie Geld zu verdienen. Dazu gibt es eine Kategorie „Business Information“, da der Streamer über seine Business-Ideen sprechen möchte und Investoren anziehen will. Weiterhin wurden auch Streams beobachtet, in denen religiöse Messen oder Zeremonien übertragen wurden, weswegen die Kategorie „Spiritualität“ verwendet wird. Folgenden Kategorien wurden für den Inhalt definiert:

Sich unterhalten, Musizieren, Informationen teilen, Nachrichten, Fitness, Sport, Gaming, Tiere, Unterhaltungsmedien, Spiritualität, Zeichnen/Malen, 24/7, Wissenschaft/ Technik/Medizin, Komödie, Alltag, Politik, Natur, Essen, Business Informationen, Nichts. 


\section{Ergebnisse}

\subsection{Demographie der Streamer}

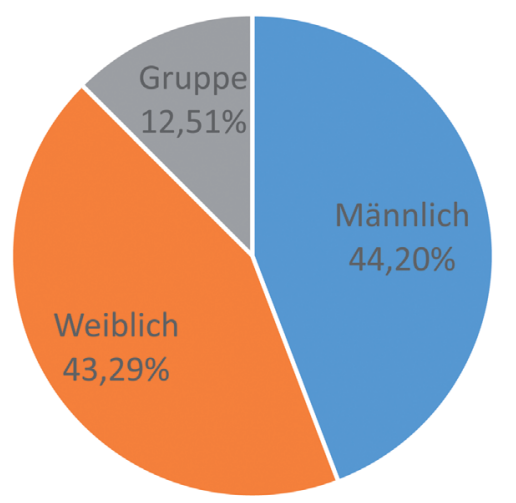

Abbildung 3: Verteilung der Geschlechter und Personengruppen bei den Streamern auf YouNow $(\mathrm{N}=1.991)$.

Die Verteilung der Geschlechter zeigt einen leicht höheren Anteil an männlichen Streamern (44,20 \%) im Vergleich zu weiblichen auf (Abb. 3). Gruppen wurden nicht in Geschlechter unterschieden und machen einen Anteil von 12,51 Prozent aus. Es wurde beobachtet, dass die Nutzer von YouNow eher alleine zu Hause vor der Webcam sitzen und sich streamen anstatt als Gruppe aufzutreten.

Die verschiedenen Generationen werden wie folgt definiert. Generation Z meint jeden, der nach 1996 geboren wurde, Generation Y zwischen 1980 und 1996, und Generation X schließt alle zwischen 1960 und 1980 Geborenen mit ein. Die Baby Boomers und Silent Generation wurden zwischen 1946 und 1960 beziehungsweise 1925 und 1945 geboren (Fietkiewicz, Lins, Baran, \& Stock, 2016).

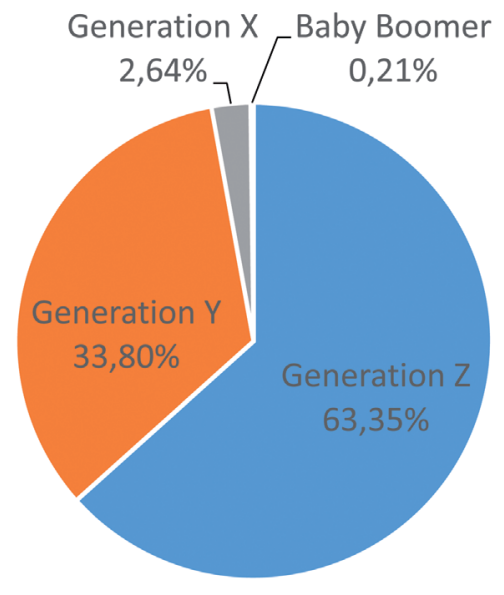

Abbildung 4: Verteilung der Generationen bei den Streamern auf Younow (N=1.932).
Die größte Nutzergruppe der Streamer auf YouNow ist die Generation Z (63,35\%) (Abb. 4). Dies beobachteten schon Honka, Frommelius, Mehlem, Tolles und Fietkiewicz (2015) sowie Scheibe, Fietkiewicz und Stock (2016). Die Nutzeranzahl sinkt stark mit zunehmendem Alter der Streamer.

\subsection{Motive}

Tabelle 1: Verteilung der Motive der Streamer auf Younow $(\mathrm{N}=2.021)$.

\begin{tabular}{llr}
\hline Rang & Motiv & Häufigkeit \\
\hline 1 & Langeweile & $35,5 \%$ \\
\hline 2 & Soziale Interaktion & $22,2 \%$ \\
\hline 3 & Spaß & $18,7 \%$ \\
\hline 4 & Selbstdarstellung & $18,3 \%$ \\
\hline 5 & Bedürfnis nach Kommunikation & $13,1 \%$ \\
\hline 6 & Meinungsaustausch & $11,8 \%$ \\
\hline 7 & Hobby & $10,7 \%$ \\
\hline 9 & Eine bestimmte Gruppe erreichen & $10,3 \%$ \\
\hline 10 & Ein Star werden & $9,7 \%$ \\
\hline 11 & Dazu gehören & $8,9 \%$ \\
\hline 12 & Beziehungspflege & $7,3 \%$ \\
\hline 13 & Selbstverbesserung & $5,7 \%$ \\
\hline 14 & Geld verdienen & $4,6 \%$ \\
\hline 15 & Einsamkeit & $3,9 \%$ \\
\hline 16 & Sendungsbewusstsein & $2,2 \%$ \\
\hline
\end{tabular}

Die Motive der Nutzer sind vielfältig (Tab. 1), doch die meisten scheinen den Service aus einfachen Gründen wie Langeweile (35,5\%) oder Spaß (18,7\%) zu nutzen. Auch Motive, die auf sozialen Bedürfnissen basieren, finden sich. Diese sind die soziale Interaktion (22,27\%), eine bestimmte Gruppe erreichen (10,3\%), dazugehören (8,9\%) und Beziehungspflege (7,3\%). Auch Gespräche untereinander sind wichtig, wie das Bedürfnis nach Kommunikation (13,1\%), Meinungsaustausch (11,8\%) oder Sendungsbewusstsein (2,2\%) zeigen. Einige Nutzer (9,7\%) verwenden YouNow, um ihre Karriere als (Micro-) Celebrity $\mathrm{zu}$ fördern und ein Star zu werden oder um Geld zu verdienen $(4,6 \%)$.

Tabelle 2 zeigt, welche Streamergruppen von YouNows Zusehern bevorzugt konsumiert werden. Hier verwenden wir die Ergebnisse unserer Online-Umfrage. 58,2 Prozent sehen am liebsten die Live-Sendungen von Freunden, gefolgt von YouTubern und neuen Broadcastern (jeweils 37,7\%). Betrachtet man Ähnlichkeiten zwischen Streamern und Viewern, bevorzugen 34,4 Prozent Strea- 
Tabelle 2: Welche Nutzergruppen bevorzugen die Zuseher? ( $\mathrm{N}=122)$.

\begin{tabular}{lr}
\hline Gruppe & Häufigkeit \\
\hline Freunde & $58,2 \%$ \\
\hline YouTuber & $37,7 \%$ \\
\hline Neue Broadcaster & $37,7 \%$ \\
\hline Gleiches Alter & $34,4 \%$ \\
\hline Gleiche Interessen & $33,6 \%$ \\
\hline Gleiches Land & $30,3 \%$ \\
\hline Gleiche Schulklasse & $7,4 \%$ \\
\hline Mädchen & $39,3 \%$ \\
\hline Jungen & $35,2 \%$ \\
\hline Alter zwischen 16 bis 20 & $42,6 \%$ \\
\hline Alter 20+ & $37,7 \%$ \\
\hline Alter zwischen 13 bis 15 & $20,5 \%$ \\
\hline
\end{tabular}

mer des gleichen Alters und 33,6 Prozent Streamer mit gleichen Interessen. Außerdem präferieren 30,3 Prozent Streamer aus demselben Land. Nur 7,4 Prozent achten bei Streamern auf die gleiche Schulklasse. Bei der geschlechterspezifischen Verteilung zeigt sich, dass YouNower eher Streams von weiblichen (39,3\%) denn männlichen (35,2\%) Personen verfolgen. Bei der Altersverteilung sehen 42,6 Prozent lieber Leuten zwischen 16 bis 20 Jahren zu. 37,7 Prozent beobachteten gerne Streams von Personen älter als 20 Jahre, und 20,5 Prozent von Teenagern (zw. 13-15 Jahre).

\subsection{Inhalt}

Streamer, die ein Livevideo starten, bereiten sich auf den Inhalt des Livestreams vor (Tab. 3). Von unseren befragten streamenden Personen gaben 80 Prozent an, vor dem streamen das Equipment zu kontrollieren, 63,3 Prozent informieren ihre Freunde und Fans und 41,7 Prozent stylen bzw. schminken sich. Themen, über die im Livestream geredet wird, bereiten 30 Prozent vor; 11,7 Prozent führen ein Stimmtraining aus.

Tabelle 3: Vorbereitung von Streamern auf einen Livestream $(\mathrm{N}=63)$.

\begin{tabular}{lr}
\hline Aktion & Häufigkeit \\
\hline Das Equipment kontrollieren & $80,0 \%$ \\
\hline Freunde und Fans informieren & $63,3 \%$ \\
\hline Sich stylen und schminken & $41,7 \%$ \\
\hline Themen vorbereiten & $30,0 \%$ \\
\hline Stimmtraining & $11,7 \%$ \\
\hline
\end{tabular}

Die meisten Videos (66,90 \%) zeigen Streamer, die sich mit den Zuschauern unterhalten oder (19,74\%) Informationen austauschen (Tab. 4). Dies beobachteten schon Tang, Veniola, und Inkpen (2016, S. 4773). Jedoch fanden sich auch 19,50 Prozent der Streams, die gar nichts zeigten. Der Inhalt weist einen interessanten Trend auf. Die sechs häufigsten Kategorien sind einfach zu produzieren und erfordern recht wenig kognitive Arbeit (sich unterhalten, Informationen teilen, Alltag, Unterhaltungsmedien). Die seltener produzierten Videos verlangen mehr Zeitaufwand, Ressourcen, Vorbereitung und kognitive Arbeit wie zum Beispiel Fitness (2,28\%) oder Wissenschaft/Technik/ Medizin $(0,25 \%)$. Die meisten Streamproduktionen erfordern wenig kognitive Anstrengung (Tyler, Hertel, McCallum, \& Ellis, 1979).

Tabelle 4: Verteilung der Inhalte auf YouNow $(\mathrm{N}=2.021)$.

\begin{tabular}{llr}
\hline Rang & Inhalt & Häufigkeit \\
\hline 1 & Sich unterhalten & $66,9 \%$ \\
\hline 2 & Informationen austauschen & $19,7 \%$ \\
\hline 3 & Nichts & $19,5 \%$ \\
\hline 5 & Musik machen & $15,7 \%$ \\
\hline 6 & Alltag & $10,2 \%$ \\
\hline 7 & Unterhaltungsmedien & $9,1 \%$ \\
\hline 8 & Komödie & $3,3 \%$ \\
\hline 9 & Gaming & $3,1 \%$ \\
\hline 10 & Fitness & $2,3 \%$ \\
\hline 11 & Werbung & $2,0 \%$ \\
\hline 12 & Sport & $1,0 \%$ \\
\hline 13 & Tiere & $0,8 \%$ \\
\hline 14 & Zeichnen/Malen & $0,8 \%$ \\
\hline 15 & Nature & $0,7 \%$ \\
\hline 16 & Spiritualität & $0,6 \%$ \\
\hline 17 & Nachrichten & $0,5 \%$ \\
\hline 18 & Business Information & $0,4 \%$ \\
\hline 19 & Politik & $0,4 \%$ \\
\hline 20 & Wissenschaft, Technik, Medizin & $0,35 \%$ \\
\hline & $24 / 7$ & $0,2 \%$ \\
\hline
\end{tabular}

\subsection{Rechtsverletzungen}

Insgesamt waren 264 der beobachteten 2.021 Streams (also $13,1 \%$ von Rechtsverletzungen und missachteten Geschäftsbedingungen betroffen. Eine frühere Untersuchung, die Rechtsverletzungen auf SLSSs, namentlich Ustream, Periscope und YouNow verglich, ergab, dass YouNow da- 
von am wenigsten betroffen ist. Die größte Anzahl fand sich auf Ustream (20,6\%), gefolgt von Periscope (18,7\%) und mit 13,1 Prozent auf YouNow (Zimmer, Fietkiewicz, \& Stock, 2017). Am häufigsten wird bei YouNow das Musikurheberrecht verletzt (81,8\%), gefolgt vom missachteten Mindestalter $(6,4 \%)$ und Beleidigungen (3,4\%) der Zuschauer (Tab. 5). Da Musik in der Studie vielfach als Beiwerk betrachtet wurde, wäre die Anzahl der Urheberrechtsverletzungen noch höher ausgefallen, weil sehr viele Streamer die Musik im Hintergrund laufen ließen.

Tabelle 5: Verteilung der Rechtsverletzungen auf YouNow.

\begin{tabular}{lr}
\hline Alle Streams $(\mathbf{N}=\mathbf{2 . 0 2 1})$ & 264 \\
\hline Rechtsverletzungen & $13,1 \%$ \\
\hline Relative Häufigkeit & \\
\hline Streams mit Rechtsverletzungen $(\mathbf{N}=\mathbf{2 6 4})$ & $81,8 \%$ \\
\hline Urheberrecht - Musik & $6,4 \%$ \\
\hline Mindestalter & $3,4 \%$ \\
\hline Beleidigungen & $2,7 \%$ \\
\hline Urheberrecht - Video & $2,3 \%$ \\
\hline Persönlichkeitsrechte & $1,5 \%$ \\
\hline Straßenverkehrsordnung & $1,1 \%$ \\
\hline Datenschutz & $0,8 \%$ \\
\hline Sportübertragungsrechte & $0 \%$ \\
\hline Urheberrecht - Bilder & $0 \%$ \\
\hline HIPAA &
\end{tabular}

Da YouNow das Mindestalter der Streamer auf 13 Jahre setzt und der Service bei den jüngeren Nutzern sehr beliebt ist und auch Kinder unter 13 Jahren anzieht, überrascht es nicht, dass die Missachtung des Mindestalters die am zweithäufigste Regelübertretung ist. Das Alter spielt auch bei der Zahl der Beleidigungen eine Rolle, da die jugendlichen YouNow-Nutzer unvorsichtiger damit umgehen. Es wurden keine Übertragungen von Live-Operationen beobachtet, so kam es auch nicht zu Verletzungen des Health Insurance Portability and Accountability Acts.

\subsection{Multi-Channel-Verhalten}

Tabelle 6 zeigt, wie viele der von uns befragten Nutzer von YouNow welche anderen Social Media-Dienste mit ihrem YouNow-Auftritt verlinken. Facebook sowie Twitter werden von jeweils 55 Prozent genutzt. 45,9 Prozent der Nutzer Verlinken auch auf Instagram. Immerhin 33,3 Prozent vernetzen die Plattform YouTube, wohingegen nur zwei Prozent zu Tumblr und jeweils ein Prozent zu Snapchat oder zu Google+ verlinken. Diese weiteren Kanäle werden u.a. dafür benutzt, um Zuseher über kommende Live-Videos zu unterrichten.

Tabelle 6: Multi-Channel-Verhalten von YouNow-Nutzern. Links zu anderen Diensten $(\mathrm{N}=111)$.

\begin{tabular}{lr}
\hline Social Network & Häufigkeit \\
\hline Facebook & $55,0 \%$ \\
\hline Twitter & $55,0 \%$ \\
\hline Instagram & $45,9 \%$ \\
\hline YouTube & $33,3 \%$ \\
\hline Tumblr & $2,0 \%$ \\
\hline Snapchat & $1,0 \%$ \\
\hline Google+ & $1,0 \%$ \\
\hline
\end{tabular}

Auch haben Nutzer (Streamer wie Zuseher) von YouNow Erfahrungen im Umgang mit anderen Video- bzw. LiveStreaming-Diensten (Tab. 7): 69,2 Prozent mit YouTube, 41,8 Prozent mit Google Hangouts und 39,6 Prozent mit Twitch. Auf Ustream haben sich schon 14,3 Prozent aufgehalten und auf Paltalk 4,4 Prozent. Picarto sowie Periscope wurde von jeweils nur 3,3 Prozent, TinyChat, Omegle von jeweils 2,1 Prozent und ChatRoulette von 1,1 Prozent schon einmal genutzt.

Tabelle 7: Erfahrungen der YouNow-Nutzer mit anderen VideoDiensten $(\mathrm{N}=91)$.

\begin{tabular}{lr}
\hline Video-Dienst & Häufigkeit \\
\hline YouTube & $69,2 \%$ \\
\hline Google Hangouts & $41,8 \%$ \\
\hline Twitch & $39,6 \%$ \\
\hline Ustream & $14,3 \%$ \\
\hline Paltalk & $4,4 \%$ \\
\hline Picarto & $3,3 \%$ \\
\hline Periscope & $3,3 \%$ \\
\hline TinyChat & $2,1 \%$ \\
\hline Omegle & $2,1 \%$ \\
\hline ChatRoulette & $1,1 \%$ \\
\hline
\end{tabular}

\subsection{Einschätzung des Systems aus der Sicht der Nutzer}

Bei der Beschreibung der Nutzerakzeptanz von YouNow (Tab. 8) orientieren wir uns am Information Service Evaluation (ISE) Modell (Schumann \& Stock, 2014). Der Frage, 
ob YouNow einfach zu nutzen wäre, haben 82,8 Prozent zugestimmt. 64,7 Prozent der Nutzer haben Spaß an YouNow. Immerhin 19,7 Prozent haben keinen oder nur wenig Spaß an YouNow, nutzen den Dienst offenbar trotzdem. Für etwas mehr als die Hälfte der Befragten (54,1\%) ist YouNow ein nützlicher Dienst. Nur 34,5 Prozent der Nutzer empfinden YouNow als vertrauenswürdig; der Großteil $(55,7 \%)$ ist sogar vom Gegenteil überzeugt.

Tabelle 8: Akzeptanz der Technologie von YouNow $(\mathrm{N}=122)$.

\begin{tabular}{lrrr}
\hline Aussage & Ja & Nein & Neutral \\
\hline YouNow ... & & & \\
\hline$\ldots$ ist einfach zu nutzen & $82,8 \%$ & $9,8 \%$ & $7,4 \%$ \\
\hline$\ldots$ zu nutzen macht Spaß & $64,7 \%$ & $19,7 \%$ & $15,6 \%$ \\
\hline$\ldots$ ist nützlich & $54,1 \%$ & $29,5 \%$ & $16,4 \%$ \\
\hline$\ldots$ ist vertrauenswürdig & $34,5 \%$ & $55,7 \%$ & $9,8 \%$ \\
\hline
\end{tabular}

In Tabelle 9 sind Gründe aufgeführt, warum derzeit aktive Nutzer den Dienst YouNow in Zukunft nicht mehr nutzen würden. Mit 54,8 Prozent ist der ausschlaggebendste Grund, wenn es zu langweilig wird. Auch würden 51,6 Prozent der Befragten YouNow nicht mehr nutzen, wenn ihre persönlichen Daten missbraucht werden. Weitere Gründe YouNow zu verlassen sind, wenn sie zu alt dafür werden $(35,5 \%)$ oder wenn ihre Freunde nicht mehr dabei sind $(28,8 \%)$.

Tabelle 9: Opting out: Gründe, die Nutzung von YouNow zu beenden $(\mathrm{N}=122)$

\begin{tabular}{lr}
\hline Grund & Häufigkeit \\
\hline YouNow zu nutzen wird langweilig & $54,8 \%$ \\
\hline YouNow missbraucht meine persönlichen Daten & $51,6 \%$ \\
\hline Ich werde zu alt für YouNow & $35,5 \%$ \\
\hline Meine Freunde nutzen YouNow nicht mehr & $28,8 \%$ \\
\hline
\end{tabular}

\section{Diskussion}

Die Analyse von YouNow weist einige interessante Trends auf. Zunächst lässt sich wiederholt beobachten, dass besonders Teenager den Service verwenden und bevorzugt Streams von Gleichaltrigen und besonders den 16- bis 20Jährigen angeschaut werden. Es ist eine Plattform von Jugendlichen für Jugendliche. $\mathrm{Zu}$ beachten ist außerdem, dass mehr männliche Streamer auf YouNow vertreten sind und weibliche Streamer bevorzugt zuschauen. Hauptmotive, YouNow zu verwenden, sind Langeweile, soziale Inter- aktion und Spaß. Aber auch die Möglichkeit, mit anderen zu kommunizieren, wird sehr geschätzt. Die Inhalte gestalten sich vielfach eher simpel und benötigen keine große Vorbereitung oder kognitive Anstrengung. Dies spiegelt sich in der Art der Vorbereitung für den Stream wider, da die meisten Streamer zwar vor dem Broadcast das Equipment kontrollieren und sich stylen, aber eher wenig aufwändigere Vorkehrungen treffen, wie ein Thema vorzubereiten oder Stimmübungen zu machen. Etwa 60 Prozent der Streamer sagen ihren Freunden oder Fans Bescheid, bevor sie live gehen, denn die Nutzer gucken bevorzugt die Streams ihrer Freunde. Da die Plattform zumeist von Jugendlichen genutzt wird, spiegelt sich dies auch in den Rechtsverletzungen wider. Hier wurden besonders die Missachtung des Mindestalters und Beleidigungen beobachtet. Auch die Rechtsverletzungen hinsichtlich der Verwendung von Musik lassen sich durch den Inhalt erklären, da häufig Videos im Hintergrund, unsichtbar für den Zuseher, beobachtet wurden, in denen Musik lief, während der Streamer abwesend war. Auf YouNow wurden nur wenige Fälle beobachtet, in denen nicht sorgfältig mit den persönlichen Daten umgegangen wurde. Die Nutzer gaben an, dass ein schädlicher Umgang mit den persönlichen Daten durch YouNow für sie Grund wäre, diese Plattform nicht weiter zu verwenden. Wenn YouNow den Streamern zu langweilig werden würde, wäre dies ebenfalls ein Motiv, den Dienst nicht mehr zu nutzen; allerdings ist Langeweile auch der Hauptgrund, YouNow überhaupt zu verwenden. Die Nutzer geben an, dass sie auch andere soziale Netzwerke in ihrem Channel integrieren, hier sind Facebook und Twitter die wichtigsten Informationsdienste. Die meiste Erfahrung mit anderen Plattformen haben die Nutzer mit YouTube, Google Hangouts und Twitch. Es lassen sich interessante Rückschlüsse über die Meinung der Nutzer zur Qualität von YouNow ziehen. Die Zuschauer erwarten, dass sie verstehen, was der Streamer tut. Dies wurde jedoch nicht so häufig bestätigt. Ebenso scheinen die $\mathrm{Zu}-$ schauer zu denken, dass die Streamer darin Erfahrung haben, was sie tun. Auch dies konnte jedoch nicht so häufig beobachtet werden. Die Chat-Nachrichten werden als grammatikalisch falsch empfunden und auch scheint die Erwartung höher zu sein, den Inhalt solcher Kurztexte zu verstehen, als es letztendlich tatsächlich der Fall ist.

Wie kann man die Forschungen zum Informationsverhalten auf Social Live-Streaming Services fortführen? YouNow arbeitet mit einer Reihe von Funktionen, die Gamification einsetzen (Batches, Ranglisten usw.). Hier sollte eine Untersuchung ermitteln, ob und in welchem Umfang solche Funktionen die Motivation von Nutzern steigern, den Dienst zu adaptieren oder ständig zu nutzen. Weiterhin bietet es sich an, auch andere Social Live-Streaming 
Dienste wie Periscope, Ustream, Facebook Live oder YouTube Live auf das Informationsproduktions- und -rezeptionsverhalten der Nutzer zu analysieren. Hierbei könnte auch die Erklärungskraft unseres theoretischen Modells basierend auf Lasswell-Formel und Uses and GratificationTheorie - überprüft werden.

\section{Literatur}

Beldad, A. D., \& Koehorst, R. (2015). It's not about the risks, l'm just used to doing it: Disclosure of personal information on Facebook among adolescent Dutch users. In G. Meiselwitz (Ed.), Social Computing and Social Media. 7th International Conference, SCSM 2015 HELD as Part of HCI International 2015 Los Angeles, CA, USA, August 2-7, 2015 Proceedings. (S. 185-195). Cham, Switzerland: Springer. doi:10.1007/978-3319-20367-6_19

Blumler, J. G., \& Katz, E. (1974). The Uses of Mass Communications: Current Perspectives on Gratifications Research. Newbury Park, CA: Sage.

Brandtzæg, P. B., \& Heim, J. (2009). Why people use Social Networking Sites. In A. A. Ozok \& P. Zaphiris (Eds.), Online Communities and Social Computing. Third International Conference, OCSC 2009, Held as Part of HCI International 2009, San Diego, CA, USA, July 19-24, 2009 Proceedings. (S. 143-152). Berlin, Germany: Springer. doi:10.1007/978-3-642-02774-1_16

Cheung, C. M. K., Chiu, P. Y., \& Lee, M. K. O. (2011): Online social networks: Why do students use Facebook? Computers in Human Behavior, 27, 1337-1343. doi:10.1016/j.chb.2010.07.028

Edelmann, M. (2016). From Meerkat to Periscope: Does intellectual property law prohibit the live streaming of commercial sporting events? Columbia Journal of Law \& the Arts, 39(4), 469-495. URL: https://journals.cdrs.columbia.edu/wp-content/uploads/ sites/14/2016/06/1-39.4-Edelman.pdf

Fietkiewicz, K. J., Lins, E., Baran, K. S., \& Stock, W. G. (2016). Intergenerational comparison of social media use: Investigating the online behavior of different generational cohorts. In Proceedings of the 49th Hawaii International Conference on System Sciences (S. 3829-3838). Washington, DC: IEEE Computer Society. doi: 10.1109/HICSS. 2016.477

Fietkiewicz, K. J., \& Scheibe, K. (2017). Good morning ... good afternoon, good evening and good night: Adoption, usage and impact of the live streaming platform YouNow. In 3rd International Conference on Library and Information Science (LIS 2017). Sapporo, Japan, August 23-25, 2017.

Friedländer, M. B. (2017a). And action! Live in front of the camera: An evaluation of the social live streaming service YouNow. International Journal of Information Communication Technologies and Human Development, 9(1), 15-33. doi: 10.4018/ IJICTHD.2017010102

Friedländer, M. B. (2017b). Streamer motives and user-generated content on social live-streaming services. Journal of Information Science Theory and Practice, 5(1), 65-84. doi: 10.1633/ JISTaP.2017.5.1.5

Greenwood, D. N. (2013). Fame, Facebook, and Twitter: How attitudes about fame predict frequency and nature of social media use. Psychology in Popular Media Culture, 2(4), 222-236. doi:10.1037/ppm0000013
Gros, D., Wanner, B., Hackenholt, A., Zawadzki, P., \& Knautz, K. (2017). World of streaming. Motivation and gratification on Twitch. In G. Meiselwitz (Ed.), Social Computing and Social Media. Human Behavior (S. 44-57). Cham, Switzerland: Springer. (Lecture Notes in Computer Science; 10282). doi: 10.1007/978-3-319-58559-8_5

Hampton, K. N., Shin, I., \& Lu, W. (2016). Digital media and stress: The cost of caring 2.0. Information, Communication \& Society, 19(9), 1267-1286. doi: 10.1080/1369118X.2016.1186714

Hollenbaugh, E. E., \& Ferris, A. L. (2013). Facebook self-disclosure: Examining the role of traits, social cohesion, and motives. Computers in Human Behavior, 30, 50-58. doi: 10.1016/ j.chb.2013.07.055

Honka, A., Frommelius, N., Mehlem, A., Tolles, J. N., \& Fietkiewicz, K. J. (2015). How safe is YouNow? An empirical study on possible law Infringements in Germany and the United States. The Journal of MacroTrends in Social Science, 1(1), 1-17. URL: http://macrojour nals.com/yahoo_site_admin/assets/docs/1SS11S36202402 .pdf

Hsu, M. H., Chang, C. M., Lin, H. C., \& Lin, Y. W. (2015). Determinants of continued use of social media: The perspectives of uses and gratifications theory and perceived interactivity. Information Research, 20(2), paper 671. URL: http://InformationR.net/ir/ 20-2/paper671.html

Joinson, A. N. (2008). Looking at, looking up or keeping up with people? Motives and use of Facebook. In Proceedings of the SIGCHI Conference on Human Factors in Computing Systems (S. 1027-1036). New York, NY: ACM. doi: 10.1145/ 1357054.1357213

Katz, E., Blumler J. G., \& Gurevitch, M. (1973). Uses and gratifications research. The Public Opinion Quarterly, 37(4), 509-523

Khoo, C. S. G. (2014). Issues in information behavior on social media. Libres, 24(2), 75-+96. URL: http://www.libres-ejournal.info/wpcontent/uploads/2015/03/LIBRESv24i2p75-96.Khoo_.2014.pdf

Kim, D., Kim, J. H., \& Nam, Y. (2014). How does industry use social networking sites? An analysis of corporate dialogic uses of Facebook, Twitter, YouTube, and Linkedln by industry type. Quality \& Quantity, 48(5), 2605-2614. doi: 10.1007/s11135013-9910-9

Kim, Y., Sohn, D., \& Choi, S. M. (2010). Cultural differences in motivations for using social networking sites: A comparative study of American and Korean college students. Computers in Human Behavior, 27(1), 365-372. doi: 10.1016/j.chb.2010.08.015

Lasswell, H. D. (1948). The structure and function of communication in society. In L. Bryson (Ed.), The Communication of Ideas (pp. 37-51). New York, NY: Harper \& Brothers.

Lin, K. Y., \& Lu, H. P. (2011). Why people use social networking sites: an empirical study integrating network externalities and motivation theory. Computers in Human Behavior, 27(3), 1152-1161. doi: 10.1016/j.chb.2010.12.009

Linde, F., \& Stock, W. G. (2011). Information Markets. A Strategic Guideline for the I-Commerce. Berlin, Germany, New York, NY: De Gruyter Saur. doi:10.1002/asi.22619

McQuail, D. (1983). Mass Communication Theory. London, UK: Sage.

McQuail, D., Blumler, J. G., \& Brown, J. R. (1972). The television audience: A revised perspective. In D. McQuail (Ed.), Sociology of Mass Communications (S. 135-165). Harmondsworth, UK: Penguin.

Marwick, A. E., \& Boyd, D. (2010): I tweet honestly, I tweet passionately: Twitter users, context collapse, and the imagined 
audience. New Media \& Society, 13(1), 114-133. doi: 10.1177/ 1461444810365313

Marwick, A. E., \& Boyd, D. (2011). To see and be seen: Celebrity practice on Twitter. Convergence: The International Journal of Research into New Media Technologies, 17(2), 139-158. doi: $10.1177 / 1354856510394539$

O’Reilly, T., \& Battalle, J. (2009). Web Squared: Web 2.0 Five Years on. Abgerufen am 16. Juli 2017 von https://conferences.oreilly.com/ web2summit/web2009/public/schedule/detail/10194

Palmgreen, P., Wenner, L. A., \& Rayburn, J. D. (1980). Relations between gratifications sought and obtained: A study of television news. Communication Research, 7(2), 161-192. doi: $10.1177 / 009365028000700202$

Pötters, S. (2011). EUGH-Urteil zu Exklusivlizenzen für TV-Sportübertragungen. JuraExamen. Online Zeitschrift für Jurastudium, Staatsexamen und Referendariat. URL: http://www.juraexamen. info/eugh-urteil-zu-exklusivlizenzen-fur-tv-sportubertragun gen/

Rugg, A., \& Burroughs, B. (2016). Periscope, live-streaming and mobile video culture. In R. Lobato \& J. Meese (Eds.), Geoblocking and Global Video Culture (S. 64-73). Amsterdam, NL: Institute of Network Cultures. (Theory on Demand \#18). URL: https:// issuu.com/instituteofnetworkcultures/docs/tod_18-geoblock ing-final_cover

Scheibe, K., Fietkiewicz, K. J., \& Stock, W. G. (2016). Information behavior on social live streaming services. Journal of Information Science Theory and Practice, 4(2), 6-20. doi:10.1633/JISTaP.2016.4.2.1

Schumann, L., \& Stock, W. G. (2014). Ein umfassendes ganzheitliches Modell für Evaluation und Akzeptanzanalysen von Informationsdiensten: Das Information Service Evaluation (ISE) Modell. Information - Wissenschaft und Praxis, 65(4/5), 239-246. doi: 10.1515/iwp-2014-0043

Shao, G. (2009). Understanding the appeal of user-generated media: A uses and gratification perspective. Internet Research, 19(1), 7-25. doi: 10.1108/10662240910927795

Tang, J. C., Veniola, G., \& Inkpen, K. M. (2016). Meerkat and Periscope: I stream, you stream, apps stream for live streams. In Proceedings of the 2016 CHI Conference on Human Factors in Computing Systems (S. 4770-4780). New York, NY: ACM. doi: 10.1145/ 2858036.2858374

Toffler, A. (1980). The Third Wave. New York, NY: Marrow.

Tosun, L. P. (2012). Motives for Facebook use and expressing "true self" on the internet. Computers in Human Behavior, 28(4), 1510-1517. doi: 10.1016/j.chb.2012.03.018

Tyler, S. W., Hertel, P. T., McCallum, M. C., \& Ellis, H. C. (1979). Cognitive effort and memory. Journal of Experimental Psychology: Human Learning and Memory, 5(6), 607-617. doi: 10.1037/ 0278-7393.5.6.607

YouNow (2016). About YouNow. https://www.younow.com/about.

Zimmer, F., Fietkiewicz, K. J., \& Stock, W. G. (2017). Law infringements in social live streaming services. In T. Tryfonas (Ed.), Human Aspects of Information Security, Privacy and Trust. 5th International Conference, HAS 2017, Held as Part of HCI International 2017, Vancouver, BC, Canada, July 9-14, 2017, Proceedings (S. 567-585). Cham, Switzerland: Springer. (Lecture Notes in Computer Science; 10286). doi: 10.1007/978-3-319-58460-7_40

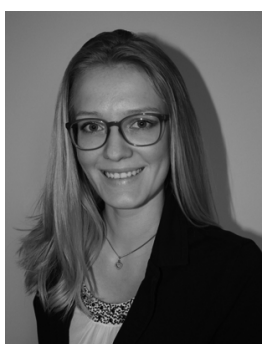

\section{Katrin Scheibe}

Heinrich-Heine-Universität Düsseldorf

Abteilung für Informationswissenschaft

Universitätsstraße 1

40225 Düsseldorf

katrin.scheibe@hhu.de

Katrin Scheibe ist wissenschaftliche Mitarbeiterin der Abteilung für Informationswissenschaft an der Heinrich-Heine-Universität Düsseldorf. Sie forscht im Themenbereich Social Media und hält Lehrveranstaltungen in den Modulen Information Retrieval sowie Informetrie.

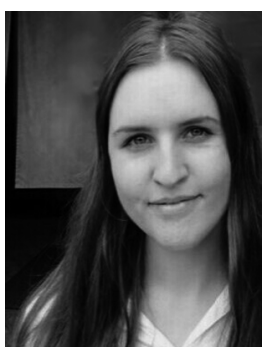

\section{Franziska Zimmer}

Heinrich-Heine-Universität Düsseldorf

Abteilung für Informationswissenschaft Universitätsstraße 1 40225 Düsseldorf franziska.zimmer@hhu.de

Franziska Zimmer ist Masterstudentin und wissenschaftliche Hilfskraft in der Abteilung für Informationswissenschaft der Heinrich-Heine-Universität Düsseldorf.

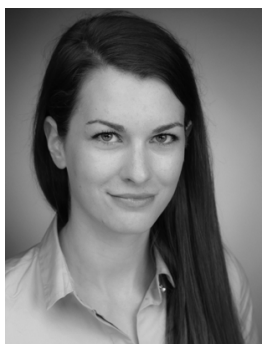

\section{Kaja J. Fietkiewicz}

Heinrich-Heine-Universität Düsseldorf

Abteilung für Informationswissenschaft Universitätsstraße 1

40225 Düsseldorf

kaja.fietkiewicz@hhu.de

Kaja J. Fietkiewicz ist Promotionsstudentin und wissenschaftliche Mitarbeiterin der Abteilung für Informationswissenschaft der Heinrich-Heine-Universität Düsseldorf. Ihre Forschungsbereiche umfassen Social Media (insbesondere Social Live Streaming Dienste), Informationsrecht und Smart City Entwicklung. 